\title{
Pneumoscrotum: a complication of pneumatosis intestinalis
}

\author{
Chirag Patel • Alex M. Barnacle
}

Received: 17 March 2010/Revised: 8 April 2010 /Accepted: 15 April 2010 /Published online: 5 June 2010

(C) Springer-Verlag 2010

A 12-year-old immunosuppressed boy presented with self-limiting abdominal pain, low-grade fever and progressive scrotal swelling. Relevant recent medical history included recurrent pneumatosis intestinalis secondary to bone marrow transplantation for relapsed non-Hodgkin lymphoma.

Abdominal radiograph (ESM Fig. 1) shows extensive bowel pneumatosis with evidence of retroperitoneal and scrotal gas. Pelvic CT (Fig. 1, CTDI =8.99 mGy) shows widespread pneumatosis intestinalis with both peritoneal and retroperitoneal gas and marked surgical emphysema extending into the perineum and scrotal wall (scrotal emphysema, long arrow). Further intra-scrotal gas appears intimately related to the right testicle (scrotal pneumatocoele, dashed arrow).

The term pneumoscrotum describes the presence of air in the scrotal soft tissues originating via one or more of three described routes [1]:

1. Scrotal emphysema: extraperitoneal air dissecting through fascial layers into the scrotal wall

2. Scrotal pneumatocoele: intraperitoneal air entering the scrotum via patent processus vaginalis [2]

3. Gas gangrene/local trauma: local production of scrotal gas/air

Electronic supplementary material The online version of this article (doi:10.1007/s00247-010-1706-7) contains supplementary material, which is available to authorized users.

C. Patel $(\bowtie) \cdot$ A. M. Barnacle

Department of Radiology,

Great Ormond Street Hospital for Sick Children,

Great Ormond Street,

London WC1N 1EH, UK

e-mail: Chiragpatel157@yahoo.co.uk
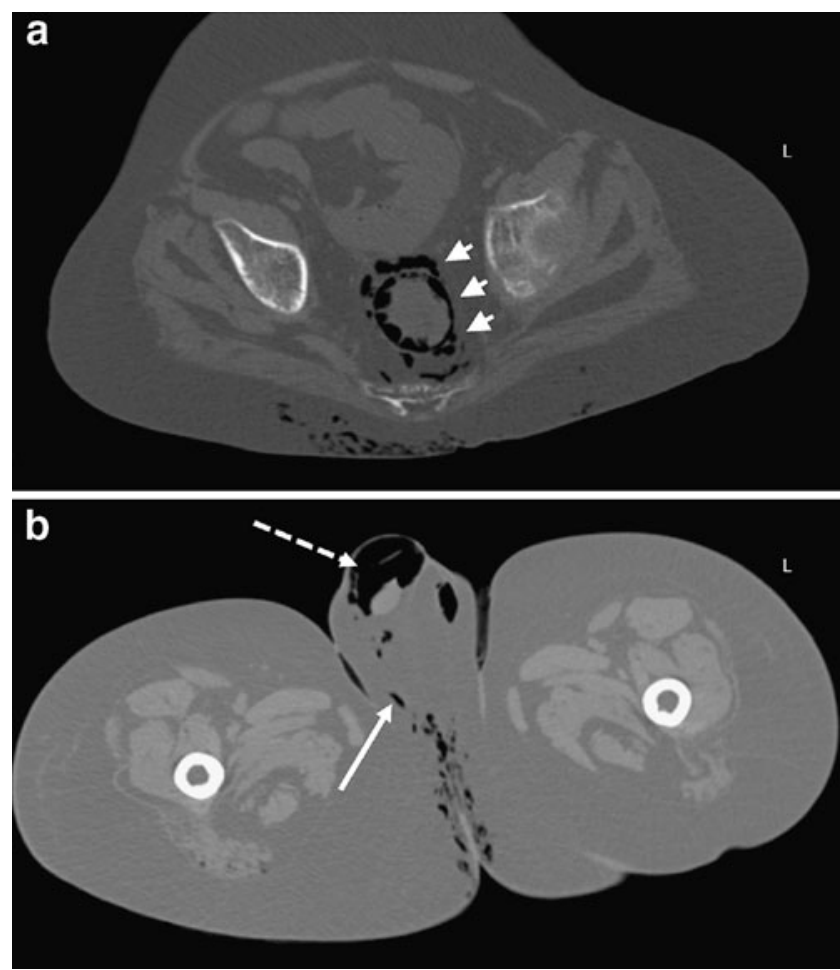

Fig. 1 Pelvic CT

\section{References}

1. Watson HS, Klugo RC, Coffield KS (1992) Pneumoscrotum: report of two cases and review of mechanism of its development. Urology 40:517-521

2. Bray JF (1982) Pneumoscrotum with testicular delineation-a new sign of pneumoperitoneum. Br J Radiol 55:867-868 